\title{
Analysis of in situ images of Bose-Einstein condensates of lithium
}

\author{
C. C. Bradley, C. A. Sackett, and R. G. Hulet \\ Physics Department and Rice Quantum Institute, Rice University, Houston, Texas 77005-1892
}

(Received 23 December 1996)

\begin{abstract}
The initial evidence for Bose-Einstein condensation (BEC) of ${ }^{7} \mathrm{Li}$ consisted of image distortions that suddenly appeared as the gas was evaporatively cooled to near the transition temperature. We present results of a model of the imaging system used to produce these images. The model confirms that the distortions are a sensitive indicator of BEC, but shows that they make quantitative analysis difficult. We find that the distortions resulted from light scattered by the condensate, in conjunction with aberrations in the imaging lens. [S1050-2947(97)05305-5]
\end{abstract}

PACS number(s): 03.75.Fi, 05.30.Jp, 32.80.Pj

In a previous Letter [1], we reported evidence for BoseEinstein condensation (BEC) of a gas of ${ }^{7} \mathrm{Li}$ atoms. In a trap, condensate atoms result in a narrow peak in the spatial density distribution. In principle, this narrow peak may be observed using an optical probe to produce absorption images of the cloud, but in our experiment the optical resolution was insufficient to directly image the condensate peak. Instead, halolike distortions surrounding the central absorption feature appeared abruptly when the gas was cooled to near the transition point, suggesting that a significant change in the spatial distribution had taken place. The appearance of these halos was originally attributed to light scattered by the condensate, and subsequently diffracted from the imaging lens aperture. In this Brief Report, we describe a model of the imaging system that shows that the distortions were indeed an indicator of the presence of a condensate, but that they were produced in conjunction with lens aberrations rather than diffraction from the aperture. Our original qualitative analysis of the images suggested a number of condensate atoms that was substantially greater than theoretical predictions [2] and our subsequently measured values [3]. The imaging model now provides a more quantitative understanding of the images, and resolves this discrepancy.

The apparatus was described in detail in previous publications $[1,4]$. Lithium atoms are confined in a permanentmagnet trap that provides a nearly isotropic harmonic trapping potential with an average oscillation frequency $\nu=135$ $\mathrm{Hz}$ [5]. Approximately $2 \times 10^{8}$ atoms were loaded into the trap, laser cooled to about $200 \mu \mathrm{K}$, and then evaporatively cooled to temperatures near $100 \mathrm{nK}$. After cooling, the trapped atom distribution was observed by in situ absorption imaging using a near-resonant optical probe. The absorptive shadow formed by the cloud in the probe laser beam was imaged onto a charge-coupled device (CCD) camera. The probe had an intensity $I=1 \mathrm{~mW} / \mathrm{cm}^{2}$, a duration of $30 \mu \mathrm{s}$, and a detuning of $\Delta=-2.5 \pm 0.5 \Gamma$, where $\Gamma=5.9 \mathrm{MHz}$ is the natural linewidth of the $2 S_{1 / 2} \leftrightarrow 2 P_{3 / 2}$ transition.

Calculated atom distributions were fit to the image data to obtain values for the total number of atoms $N$ and the temperature $T$. In the simple analysis employed in Ref. [1], the clouds were modeled using Boltzmann statistics, which give a Gaussian density profile. From the fitted values of $N$ and $T$, the phase-space density was estimated using $N / N_{c}$, where $N_{c}=1.202\left(k_{B} T / h \nu\right)^{3}$. For temperatures of approximately
$500 \mathrm{nK}$, these estimates indicated that the clouds were nearly quantum degenerate. When cooled to lower temperatures the images manifested the sudden appearance of a halolike region of "negative absorption." A cross section of an image exhibiting these distortions is shown in Fig. 1. Fitting the distorted images to Gaussian distributions resulted in phasespace densities implying a number of condensate atoms, $N_{0}$, as large as $10^{5}$. These values were higher than expected, since in our experiment, interatomic interactions were predicted to limit the condensate number to less than approximately 1400 atoms [2].

In order to understand the unexpected values of $N_{0}$ and the origin of the halos, a detailed model of the formation of images was developed. This model includes the nonclassical density distribution of the atoms, the effect of the atom cloud on the probe laser beam, and the propagation of the coher-

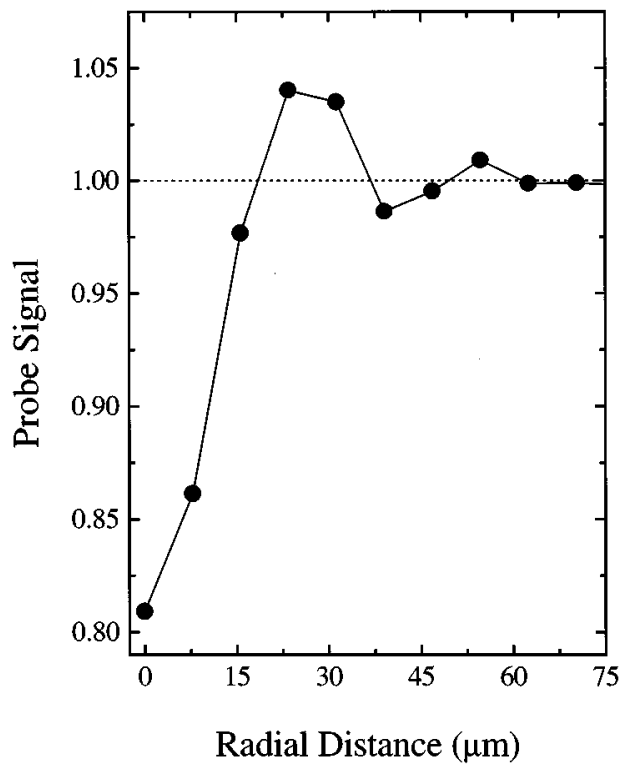

FIG. 1. Cross section taken from an absorption image of a ${ }^{7} \mathrm{Li}$ cloud, showing halolike distortions as previously described [1]. At small radii, the probe intensity is reduced due to absorption from the cloud. Near radii of about $30 \mu \mathrm{m}$, there is a region of "negative absorption,' where the image intensity is nearly $5 \%$ brighter than the background level. The imaging magnification is 2.4 and the CCD pixels are $19 \mu \mathrm{m} \times 19 \mu \mathrm{m}$ squares. 


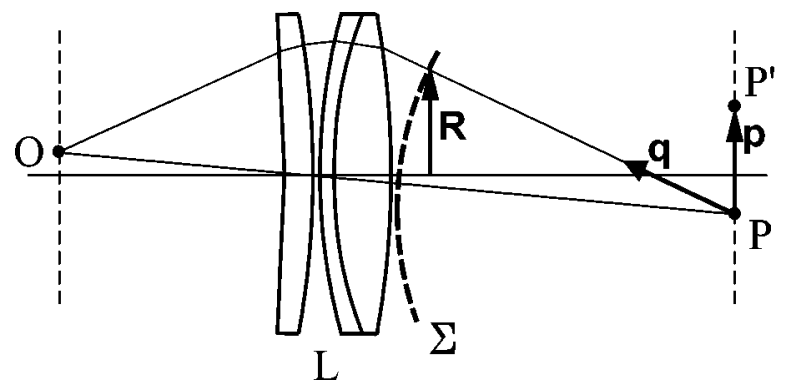

FIG. 2. Illustration showing the imaging system for the experiment of Ref. [1], for the purpose of calculating the point-transfer function. Light from an object point $O$ is collected by a set of lenses $L$ and imaged onto the image plane near the geometrical image point $P$. The displacement of the image point $P^{\prime}$ from $P$ is given by p. The surface $\Sigma$ is the exit pupil of the optics, consisting of a section of a sphere centered on $P$. A particular ray from $O$ to $P$ intersects $\Sigma$ at a position $\mathbf{R}$.

ently scattered light through the imaging optics. The incident field $E_{0}$ is considered to be uniform and to propagate along the $z$ axis. In passing through the cloud, the probe field is attenuated and acquires a phase shift. This results in an object field given by $E_{P}=E_{0} e^{i \beta}$, where $\beta$ is a spatially varying complex phase. For an atom density distribution $n(x, y, z)$, $\beta$ is given by [6]

$$
\beta(x, y)=\phi+\frac{i \alpha}{2}=-\frac{1}{2} \int \frac{n(x, y, z) \sigma_{0} \Gamma(2 \Delta-i \Gamma)}{4 \Delta^{2}+2 \Omega^{2}+\Gamma^{2}} d z,
$$

where $\alpha$ is the optical density, $\phi$ is the dispersive phase, $\sigma_{0}=\lambda^{2} / \pi$ is the resonant light scattering cross section, and $\Omega^{2}=I \sigma_{0} \Gamma / \hbar \omega$ is the square of the Rabi frequency, for transition angular frequency $\omega$ and wavelength $\lambda$. The simple analysis of Ref. [1] assumed perfect imaging, in which case the detected signal is $I_{A}(x, y)=\left|E_{P}(x, y)\right|^{2}=I_{0} e^{-\alpha(x, y)}$ and depends only on the optical density. However, the imaging system actually suffered from both a limited aperture and spherical aberration.

To include the effect of imperfect imaging in the model, the imaged field may be calculated by the convolution of the object field $E_{P}$ with the point-transfer function of the imaging system $G$. The function $G$ is the field produced at the image plane by a point source at the object plane, and is determined by analyzing the propagation of light through the imaging optics given the known lens composition and geometry. It can be written as a diffraction integral [7]:

$$
G(\mathbf{p})=C \int_{\Sigma} e^{-i k \mathbf{q} \cdot \mathbf{p}} e^{i k W(\mathbf{R})} d S
$$

The relevant quantities are illustrated in Fig. 2: $e^{i k W(\mathbf{R})}$ is the field distribution on the exit pupil $\Sigma, \mathbf{p}$ is the displacement from the central image point $P, \mathbf{q}$ is the unit vector from $P$ to a surface element $d S$ on $\Sigma$, and $\mathbf{R}$ gives the location of $d S$. The normalization $C$ and the wave number $k=\omega c$ are constants. The phase error $W(\mathbf{R})$ is the phase of the light at the exit pupil relative to a diffraction-limited spherical wave front converging on $P$. It may be estimated by tracing light rays from the object point $O$ to points on the exit pupil, taking $W(\mathbf{R})$ to be the difference in optical path length for the central ray and a ray having radius $\mathbf{R}$ on $\Sigma$.

Convolution of $E_{P}$ and $G$ is simplified for small objects located near the optical axis of the imaging system, since all object points will have approximately the same cylindrically symmetric point-transfer function. The image field $E_{I}$ at point $\mathbf{r}$ is then given by

$$
E_{I}(\mathbf{r})=\int E_{P}\left(\frac{\mathbf{r}^{\prime}}{m}\right) G\left(\mathbf{r}^{\prime}-\mathbf{r}\right) d x^{\prime} d y^{\prime}
$$

where $\mathbf{r}^{\prime}-\mathbf{r}=\mathbf{p}$ in Fig. 2. Since $E_{P}$ is calculated in the object plane, it is scaled by the magnification $m$. In the experiment, the optical system consisted of a 3-cm-diam doublet and a matching meniscus lens [8] located approximately $16 \mathrm{~cm}$ from the atom cloud. The phase error was calculated using the known lens specifications, and found to have the form $W(R)=B_{2} R^{2}+B_{4} R^{4}+B_{6} R^{6}$, with $B_{4}=-0.875 \lambda \mathrm{cm}^{-4}$ and $B_{6}=0.0066 \lambda \mathrm{cm}^{-6}$. The value of $B_{2}$ depends on the choice of image distance. At the paraxial focus $B_{2}=0$, but the optimum focal position will be different because the $R^{4}$ term, corresponding to negative spherical aberration, causes the marginal rays to focus further from the lens than the paraxial rays. Experimentally, the optics were focused using incoherent light obtained by illuminating a $200-\mu \mathrm{K}$ cloud with a set of transverse laser beams. The image distance was adjusted to give the highest peak intensity on the camera. This choice of focus is reproduced in the model at the value $B_{2}=2.5 \lambda \mathrm{cm}^{-2}$.

Because the atoms scatter photons during the probe pulse, the imaging model incorporates a time-dependent density distribution. The density is initially given by an equilibrium Bose-Einstein distribution [9], but evolves due to the heating caused by the probe. The value of the detuning was also adjusted to account for the changing Doppler shift of the accelerating atoms. The resulting instantaneous profiles are then averaged over time to give the final image.

Using the model, absorption images are generated and compared to the data. Calculated image profiles are shown in Fig. 3 for distributions with a temperature of $150 \mathrm{nK}$ and various condensate populations. Comparison with Fig. 2 shows that the curve for $N_{0}=1500$ atoms is in reasonable agreement with the data, demonstrating that the original data were consistent with a limited condensate number when imperfect imaging is taken into account.

In order to explain how the phase-space density can be overestimated by fitting with a simple Gaussian, model image profiles were calculated for particular values of $N$ and $T$, and subjected to the same analysis used in Ref. [1]. For profiles calculated from degenerate distributions, significant overestimates occur. As an example, the profile with $N_{0}=1500$ in Fig. 3 corresponds to $N / N_{c}=1.1$, but results in $N / N_{c} \simeq 7$ when fitted by a Gaussian. This effect occurs because the imaging distortions produce profiles that appear narrower (colder) and stronger (denser) than would be observed with perfect imaging.

The halos observed in the images arise from lens aberrations that cause different regions of the object field $E_{P}$ to overlap and interfere in the image plane. This interference 


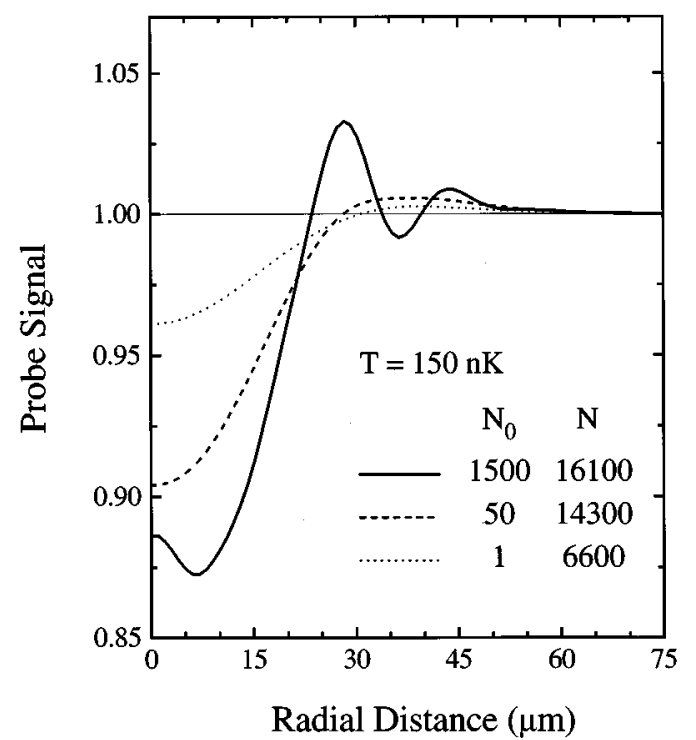

FIG. 3. Modeled absorption image profiles of Bose-Einstein distributions of trapped atoms, for the imaging system of Ref. [1]. The profiles are for 150-nK distributions with condensate numbers $N_{0}$ and total numbers $N$ as shown. The probe detuning is $2 \Gamma$. Image distortions increase sharply as atoms are added to the condensate, and appear as halos surrounding the central absorption feature. These image profiles are obtained by a convolution of the calculated object field $E_{0} \exp (i \beta)$ with imaging system point-transfer function. The calculation includes heating of the cloud due to the probe.

makes the image sensitive to spatial variations in the phase of $E_{P}$. Because the spatially small condensate produces a large phase shift, its presence can greatly enhance the distortion of the profiles. The aberrations of the imaging system are, in fact, such that there is a strong correlation between halo strength and condensate number. To show this connection, the halo intensity is plotted in Fig. 4 as a function of $N / N_{c}$, for $T=150 \mathrm{nK}$ and $\Delta=-2 \Gamma$. The figure demonstrates that aberrations result in only a weak halo for nondegenerate clouds. As the condensate forms, the object field changes dramatically, and there is a corresponding sharp rise in the halo strength.

In principle, the density distribution can be determined even for imperfect resolution, as long as the coherent pointtransfer function $G$ is known. In practice, however, quanti-

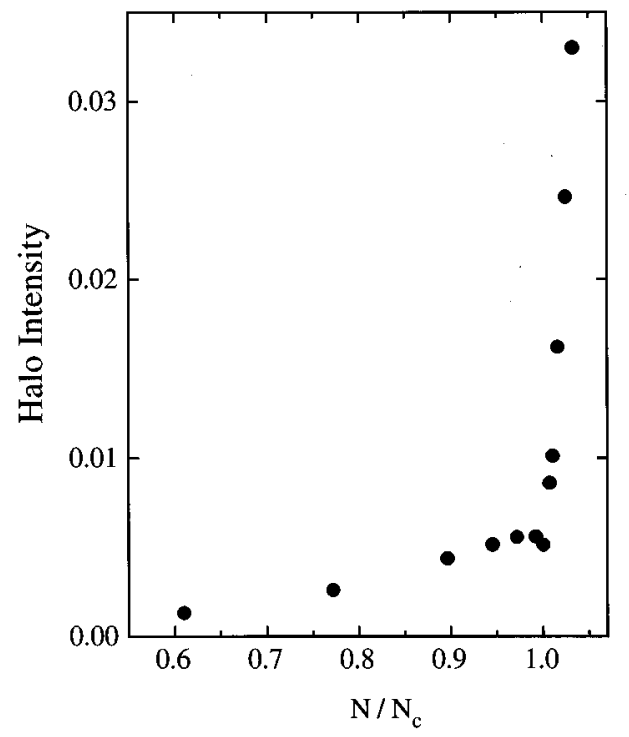

FIG. 4. The peak intensity of halo distortions for calculated images of $150-n K$ clouds as a function of the number of atoms in the cloud. The BEC phase transition is seen to be correlated with a sharp rise in the halo intensity. The halo intensity is given relative to the background probe intensity. At this temperature $N_{c}$ is $1.5 \times 10^{4}$ atoms, and the point with the largest number of atoms corresponds to $N_{0}=1500$.

tative analysis is impaired by uncertainty in $G$, largely due to the uncertainty in the experimental object and image distances. Therefore, we have recently replaced the imaging system with one having much smaller aberrations. Furthermore, we have developed a phase-contrast imaging technique that minimizes the type of distortions described in this paper, as it is sensitive to small dispersive phase shifts attained for large probe detunings. Under identical experimental conditions in which halos were originally observed, the new high-resolution images directly reveal ${ }^{7} \mathrm{Li}$ condensates [3]. Using these images, quantitative analysis indicates a maximum number of $1050 \pm 300$ condensate atoms, in reasonable agreement with the theoretical limit.

We are grateful to Mark Welling for his comments on the manuscript. This work was supported by the National Science Foundation and the Welch Foundation.
[1] C. C. Bradley, C. A. Sackett, J. J. Tollett, and R. G. Hulet, Phys. Rev. Lett. 75, 1687 (1995).

[2] P. A. Ruprecht, M. J. Holland, K. Burnett, and M. Edwards, Phys. Rev. A 51, 4704 (1995); M. Houbiers and H. T. C. Stoof, ibid. 54, 5055 (1996).

[3] C. C. Bradley, C. A. Sackett, and R. G. Hulet, Phys. Rev. Lett. 78, 985 (1997).

[4] J. J. Tollett, C. C. Bradley, C. A. Sackett, and R. G. Hulet, Phys. Rev. A 51, R22 (1995).

[5] The trap frequencies are more precisely known than in Ref.
[1], since they were subsequently directly measured.

[6] P. Meystre and M. Sargent III, Elements of Quantum Optics, 2nd ed. (Springer-Verlag, Berlin, 1991).

[7] M. Born and E. Wolf, Principles of Optics (Pergamon Press, New York, 1959).

[8] Melles-Griot lenses 01LAM225 and 01LAO225 were used. Specifications for these lenses were obtained by personal communication with technical support staff.

[9] V. Bagnato, D. E. Pritchard, and D. Kleppner, Phys. Rev. A 35, 4354 (1987). 